\title{
Molecular dynamics simulation for modeling plasma spectroscopy
}

\author{
B Talint国, E Dufour†, A Calisti†, M A Gigososț, M A

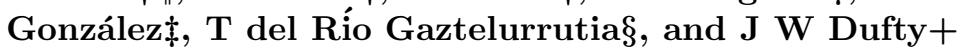 \\ † Université de Provence, CNRS UMR 6633, Centre Saint Jérôme, 13397 \\ Marseille Cedex 20, France \\ $\ddagger$ Departamento de Óptica y Física Aplicada, Facultad de Ciencias, Universidad \\ de Valladolid, 47071 Valladolid, Spain \\ $\S$ Fisika Aplikatua Saila, Industri eta Telekomunikazio Ingeniarien Goi Eskola \\ Teknikoa, Urkijo Zumarkalea z/g, S-48013 Bilbo Spain \\ + Department of Physics, University of Florida, Gainesville, FL 32611, USA
}

\begin{abstract}
The ion-electron coupling properties for a ion impurity in an electron gas and for a two component plasma are carried out on the basis of a regularized electron-ion potential removing the short-range Coulomb divergence. This work is largely motivated by the study of radiator dipole relaxation in plasmas which makes a real link between models and experiments. Current radiative property models for plasmas include single electron collisions neglecting charge-charge correlations within the classical quasi-particle approach commonly used in this field. The dipole relaxation simulation based on electron-ion molecular dynamics proposed here will provide means to benchmark and improve model developments. Benefiting from a detailed study of a single ion imbedded in an electron plasma, the challenging two-component ion-electron molecular dynamics simulations are proven accurate. They open new possibilities to obtain reference lineshape data.

PACS numbers: 52.65.Yy, 32.70.Jz, 52.27.Aj
\end{abstract}

ๆ To whom correspondence should be addressed (btalin@piima1.univ-mrs.fr) 


\section{Introduction}

In this paper classical ion-electron plasma models are used to carry out studies preliminary to line spectra simulations for ion emitters. A classical plasma model involves point charges, sometimes in a uniform charge background, interacting through given additive binary forces and parameters characterizing densities and temperatures. It is assumed that quantum mechanics are either negligible or can be incorporated through appropriate modification of the classical Coulomb potential. An example of such a classical plasma is the one component plasma (OCP) developed to study the statistical properties of non degenerate ion fluid. The present study deals with ions of charge $Z e$ and electrons. The inclusion of electrons requires essential quantum mechanical effects to eliminate the divergence of the attractive ion-electron Coulomb potential in the classical plasma. This is accomplished by using a regularized Coulomb potential which remains finite at short distances, representing quantum diffraction effects [1], and allowing the derivation of all the desired properties using the laws of classical mechanics. A detailed study of the time independent statistical properties of an electron gas with an ion impurity has been carried out recently on the basis of such a regularized electron-ion potential [2]. Two standard classical methods have been used for this study: molecular dynamics (MD) and hypernetted chain approximation (HNC) allowing to perform both cross comparisons and interpretations of the results. A first interpretation of the dynamical properties of the electron field autocorrelation function at the impurity is reported in this issue [3]. Beside structure and correlation functions MD can provide realistic representations of these stochastic electric forces at the ion. Time dependent electric fields are the necessary ingredients to describe Stark broadening (dipole relaxation) of spectral lines emitted by ions in plasmas. It is of great interest to describe these fields by simulation in order to obtain lineshapes accounting for all the correlations between charged particles. Such simulated lineshapes would provide essential reference data to benchmark more efficient phenomenological lineshape models developed for plasma diagnostics via spectroscopy. Several recent models for line broadening have been developed for investigations at new plasma conditions. They suggest that part of the discrepancies found with experiments is an inadequate description of the ion and electron perturber coupling mechanisms. The main objective of our program is to perform MD simulations with ions of charge $Z \geq 1$ and electrons in order to derive all the relevant data required for lineshape simulation accounting for all the interactions between charges. The parameter range explored in this work is chosen to be compatible with hot and dense plasmas diagnostics based on spectroscopy. This is complementary to other MD simulation studies of dense hydrogen [4. 5] with $Z=1$ and a much larger electron-electron coupling strength. Two examples are provided to show the potential for application of MD simulation to spectral line broadening. In the next two sections the effects of electron-electron correlations on electron broadening of spectral lines is studied for a massive radiator in an electron gas. All ion broadening is neglected in order to better isolate the electron coupling effects. Next, in section 4 the more realistic case of a radiator in a two component plasma (TCP) of electrons and ions is considered. It is shown that separating the effects of electron and ion broadening, as done in most current theories, requires some care and interpretation. 


\section{Ion impurity in an electron gas}

In this and the following section we consider a single ion impurity of infinite mass imbedded in an electron gas with an additional uniform positive background for charge neutrality. As mentioned above a more detailed study has been reported elsewhere so only a few remarks will be recalled here. The main statistical properties at the ion are derived using both MD simulation and HNC approximation. The ion-electron Coulomb regularized potential postulated is:

$$
V_{i e}(r)=-Z e^{2}\left(1-e^{-r / \delta}\right) e^{-r / \lambda} / r
$$

where $\delta=\left(2 \pi \hbar^{2} / m_{e} k_{B} T\right)^{1 / 2}$ is the De Broglie wavelength and $\lambda$ is a decay length long enough to represent Coulomb correlations but short enough to allow periodic boundary conditions (see below). Other parameters are the average electron-electron distance $r_{0}=\left(3 / 4 \pi N_{e}\right)^{1 / 3}$ defined in terms of the electron density $N_{e}$, the electronelectron coupling constant $\Gamma$, the electron plasma frequency $\omega_{p}$, and the ion-electron potential at the origin which, in units of $r_{0}$, becomes: $\sigma=Z \Gamma / \delta$. For simplicity, the OCP electron-electron potential is taken to be $V_{e e}(r)=e^{2} e^{-r / \lambda} / r$.

Molecular dynamics simulations are carried out using a number $N$ of electrons in the elementary cubic cell of size $c$ large enough to ensure the following constraints: $Z<<N, \lambda>\lambda_{D}$ where $\lambda_{D}$ is the Debye wavelength and $\lambda \leq c / 2$. These conditions are required in order that the Coulomb screening mechanisms actually reduce the ionion interaction length.

The hypernetted chain set of equations [6] is written for the single ion case, i.e. for a vanishing ion density. As a result, the ion-electron pair distribution function $g_{i e}(r)$ is obtained by iteration as a function of the OCP direct electron-electron correlation function $c_{e e}(r)$ calculated separately. Both MD simulation and HNC approximation are restricted to a limited range of values for the ion charge, density, and temperature. Outside of this range electron trapping and non-convergent iteration could occur invalidating the results. For the parameter space considered MD and HNC results are in good agreement as illustrated in Fig 1. The electron accretion around the ion impurity shown by the shape of the ion-electron pair distribution function is the expression of screening mechanisms taking place due to the attractive forces. It will be indirectly shown later that for these plasma parameters the screening length is the Debye length.

\section{Implication for dipole relaxation mechanisms}

Most frequently models for Stark effect in plasmas are semiclassical, i.e. they rely on the hypothesis that line broadening can be given by the evolution of a quantum system - the emitter - perturbed by the classical plasma electric micro-field. In addition it is supposed that the emitter size is small in respect to the micro-field space fluctuation so that this coupling occurs via dipole interaction with the total electric micro-field of the plasma. Due to the small electron-ion mass ratio, the Stark effect resulting from slow ion and fast electron micro-fields are generally considered separately. In this way the ion Stark effect is approximated by a static field model, while electron broadening is described as a dynamical process resulting from the high frequency stochastic fluctuation of the electron micro-field. In order to illustrate the implication of the present study for line broadening attention is restricted in this section to electron broadening alone, using the simple case of a massive emitter in an 


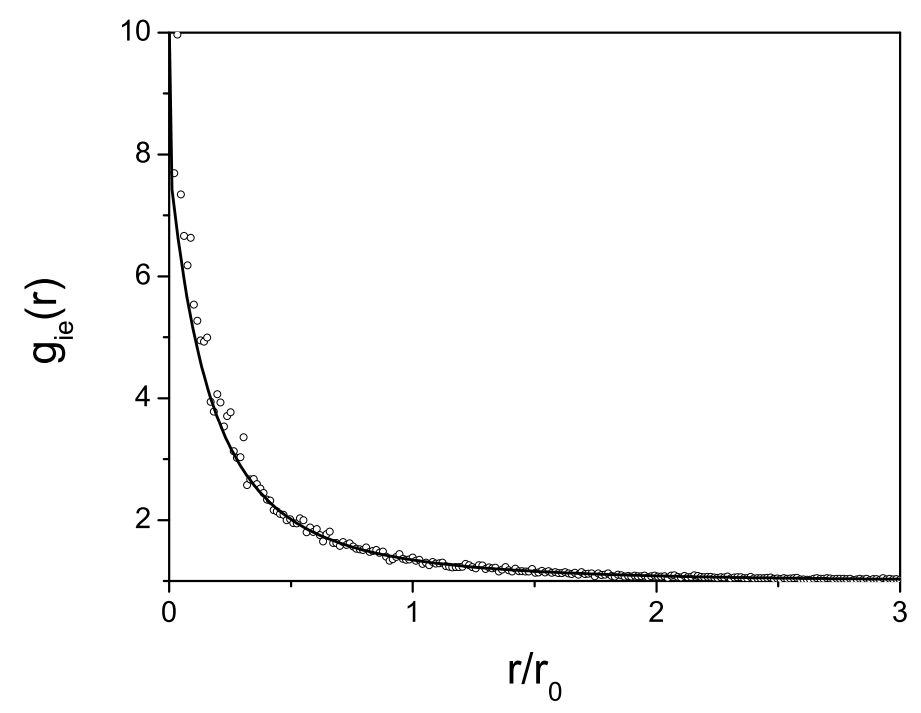

Figure 1. CVI impurity ion-electron pair correlation function: $Z=5, N_{e}=$ $10^{21} \mathrm{~cm}^{-3}$ and $T_{e}=232000^{0} \mathrm{~K}$ from HNC: solid line, MD: circles

electron gas. The usual approach for electron broadening for ion emitters in plasmas relies on a binary collision model involving independent electrons moving on straight trajectories at constant velocity. Electron correlations are accounted for indirectly by screening the electron-emitter potential at the Debye length. The discussion of this approximation is not the purpose here, but rather it is mentioned to motivate the following comparison. Two MD simulations are performed to calculate the electron electric field at the emitter, followed by calculation of the spectral line shape: 1) the electrons are considered as free particles that do not interact with each other but perturb the ion emitter with a field obtained from the potential written in (1) with $\left.\lambda=\lambda_{D} ; 2\right)$ the electrons have Coulomb interactions with each other and couple to the emitter with a field obtained using in (11) with $\lambda=c / 2$. The latter is, of course, the correct treatment of correlations. Given the fields from these MD calculations a lineshape simulation follows. Although generally not coded in that way this simulation process is equivalent, first, to solve numerically a stochastic equation for the quantum system evolution operator:

$$
\begin{aligned}
& \frac{d U_{f_{i}}(t)}{d t}=-i L_{f_{i}}(t) U_{f_{i}}(t) \\
& \mathbf{U}(t)=\left\{U_{f_{i}}(t)\right\}_{a v}=\frac{1}{N} \sum_{i=1}^{N} U_{f_{i}}(t)
\end{aligned}
$$

where $\left\{f_{1}, f_{2} \ldots f_{N}\right\}$ is a sample set of independent perturbing field histories and $L_{f_{i}}(t)$ is an operator involving the coupling of the emitter dipole with the external field $f_{i}$. Then, the lineshape results from Fourier transform of the emitter dipole autocorrelation function. It is written in Liouville space as a scalar product in terms 


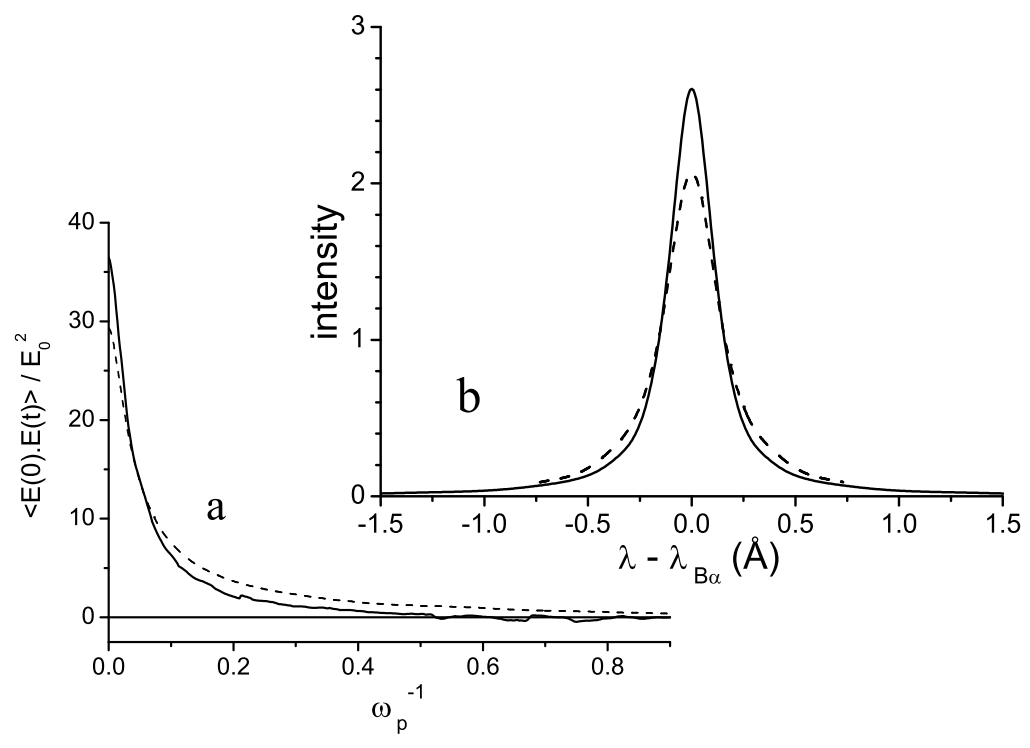

Figure 2. HeII impurity for independent electrons (dashed line) and coupled electron (solid line): $Z=1, N_{e}=10^{18} \mathrm{~cm}^{-3}$ and $T_{e}=60000 \mathrm{~K}$. a) field autocorrelation function, b) Balmer alpha line

of the dipole vector $|\mathbf{d}\rangle\rangle$ and the equilibrium density matrix $\rho$ :

$$
I(\omega)=\frac{1}{\pi} \int_{0}^{\infty}\langle\langle\mathbf{d}|\mathbf{U}(t)| \rho \mathbf{d}\rangle\rangle d t
$$

These simulations require a fast integration process based on an earlier work [7] carried out for hydrogenic lines. The example reported is the Balmer alpha line emitted by an hydrogen-like helium emitter in an electron gas with the density $N_{e}=10^{18} \mathrm{~cm}^{-3}$ and the temperature $T_{e}=60000 \mathrm{~K}$. Figure 2 shows comparisons of independent electrons (case 1) and coupled electrons (case 2). From the field autocorrelation functions plotted on Fig 2-a $\langle\mathbf{E}(t) \cdot \mathbf{E}(0)\rangle / E_{0}^{2}$, where brackets indicate a classical Gibbs ensemble average and $E_{0}$ is the mean electronic field, it can be inferred that the field de-correlation rate of case 1 is lower than for case 2. An alternative interpretation is that the field fluctuation rate is larger when charge-charge correlations are accounted for than if they are not. As the broadening mechanism is mainly a dynamical process, a larger field fluctuation rate results in a narrower line, as shown on Fig. 2b. However, this behavior found for an ionic charge $Z=1$ cannot be generalized straightforwardly since the broadening effect depends not only on the field fluctuation rate but also on the field covariance which is an increasing function of $Z$.

\section{Two component plasma MD simulations}

Intuitively, the electron microfield properties investigated above for a single ion in an electron gas will be different if the positive background assumed for charge neutrality is replaced by mobile ions. In this section a charge-neutral two-component plasma 
(TCP) of electrons and ions of charge $Z e$ will be considered. Classical MD simulation is used to account for correlations among all charges and cross comparisons performed for the single ion case ensure the accuracy of these TCP simulations. The challenging aspect of this study is to move slow and fast particles at the same time. This requires simulations stable over a long period of time, long enough to account for ion motion and based on a time step compatible with electron motion. These simulations are expensive as the particle number in the elementary cubic cell, $N_{i}$ ions and $Z N_{i}$ electron, is large. However, a few simulations have been carried out successfully. That for the same plasma conditions than in Fig 1 is reported here. Before proceeding it is worth contrasting such a MD simulation with other methods of describing the TCP. Simulations involving ions and electrons of a two component plasma have been carried out with the so called quantum molecular dynamics [8, 9]. At each time step the electrons are equilibrated about the ion configuration (Born-Openheimer approximation) using an appropriate density functional model. The ion-ion forces are then calculated from the resulting electron density and these forces are used in classical MD to move the ions. The process is repeated at each time step. Alternatively, the electrons can be equilibrated by the Carr-Parinello method. In both cases the electron dynamics is fully equilibrated at each time step. The advantage of MD simulation, although limited by the semi-classical potential, is that both electrons and ions move dynamically according to the given equations of motion. It appears that the two classes of methods are complementary, with quantum molecular dynamics being more appropriate for lower temperature and MD more appropriate at higher temperatures.

\subsection{Screening effects}

The first point of interest is to verify that the expected ion-electron screening mechanisms take place properly. As noted before, in the MD elementary cubic cell the charge-charge interaction length is half the cell size while the expected effective screening length (Debye) is only a fraction of it. A comparison of the ion-ion pair distribution function for the TCP and for the corresponding ion OCP is made. In the OCP system the ion-ion Coulomb potential is screened at the Debye length, while such screening must be generated by the electrons in the TCP simulations. Figure 3 shows a good agreement between the two simulations giving an indirect evidence of the effectiveness of the ion-ion screening by the electrons. Other calculations not reported here show that the agreement is preserved as well when the temperature varies.

\subsection{Field dynamical properties}

Molecular dynamics simulation allows determination of statistical data for each kind of particle involved. In the electron-ion TCP two kinds of time dependencies coexist, the high frequency and the low frequency dynamics related to electron motion and ion motion, respectively. Both of them are of interest for probing or discussing the simulation accuracy. For the high frequency data the electron field autocorrelation function (FAF) at the ions has been calculated. Figure 4-a shows a comparison of the field autocorrelation function for a single ion in electrons and for the TCP. It can be observed that for the TCP the FAF does not go to 0 as for the impurity case but, at the high frequency time scale used for Fig 4-a, keeps a constant value that denotes the occurrence of a low-frequency component in the electron field. At 


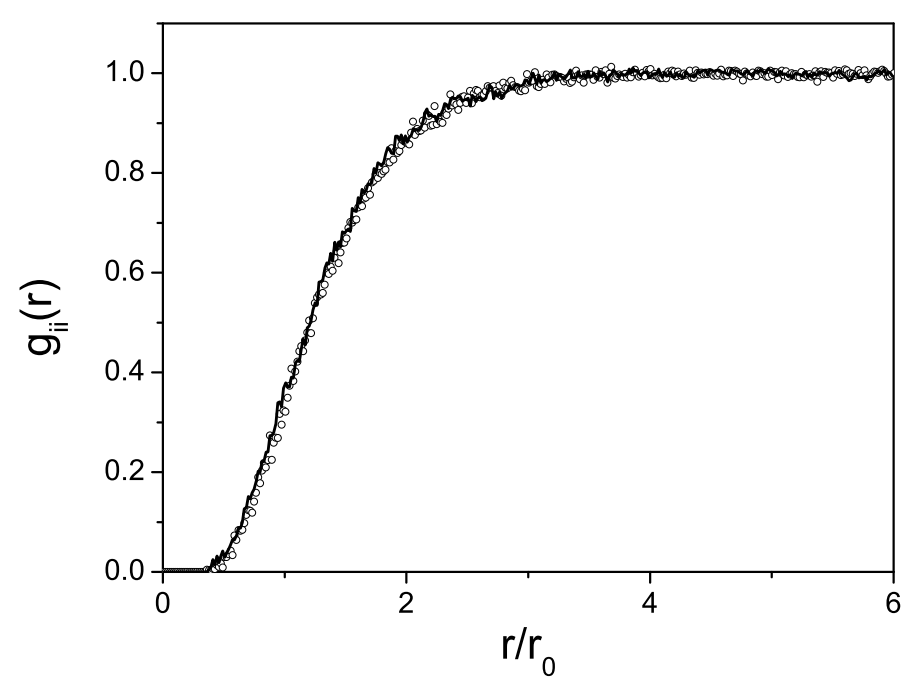

Figure 3. CVI ion-ion pair correlation function, same conditions as in Fig 1; TCP: solid line, ion OCP: circles

a low-frequency dynamics time scale it will be seen below that, as expected, this constant value goes to 0 . The low-frequency component can be obtained averaging the electron field at the ions on a period of time long enough for the field to loose correlation of the high frequency fluctuation. Contrary to the single ion case, a given ion is no longer a center of symmetry for the smooth electron density that could result from the same high frequency dynamics time average. Due to the screening mechanisms, the structure of this smooth electron density is a blurred image of the ion configuration which remains quasi-static at this time scale. The primary consequence of the occurrence of a low-frequency component in the TCP electron field is that it can no longer be used in lineshape simulations. This will be a motivation to carry out lineshape simulations accounting for the total ion plus electron field. In order to investigate how the low-frequency component is driven by the ion configuration several FAF have been plotted on Fig 4-b: 1) the field autocorrelation function of the low-frequency component, labelled "electron TCP"; 2) the ion FAF for the ion OCP; 3) the ion FAF for the TCP. It can be noted that the low-frequency component FAF goes to 0 with nearly the same rate than the ion FAF curves. For the TCP the ion-ion interaction length is the cell size while for the OCP it is the Debye length. This induces an inadequacy of the actual ion-ion coupling and the pure ion-ion field. Therefore, the ion TCP field appears useless when considered without the screening electron field i.e. without the low-frequency component. This explains the quite large discrepancy for the initial value of the two ion FAF curves. Some differences occur also between the dynamical behavior of the various partial fields. Further results and analysis will be given elsewhere. 


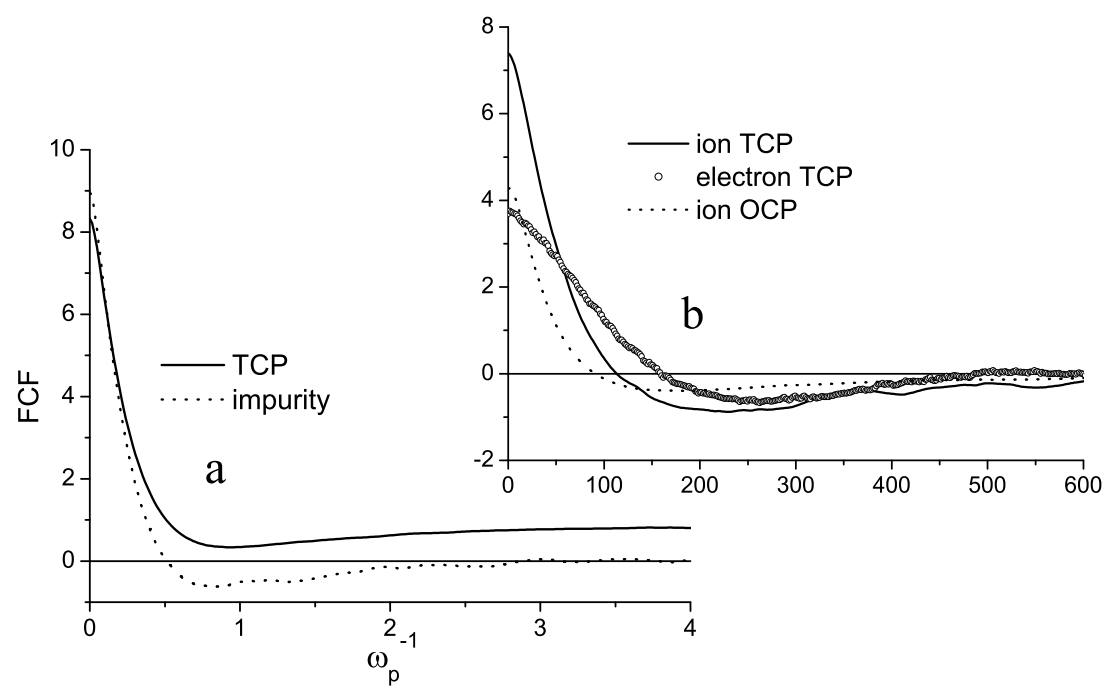

Figure 4. (same as in Fig 1 and 3) a) electron FAF: single ion case and TCP, b) time averaged electron $\times 5$, ion OCP and ion TCP field auto correlation functions

\section{Discussion}

Classical molecular dynamics simulations of a two component electron-ion plasma based on a regularized potential have been carried out successfully. The step by step motion of slow ions and fast electrons considered at the same time is inherently time consuming. However, MD is a unique way for providing static and time dependent statistical data accounting for all the charge-charge couplings. Among them, the screening mechanisms of forces take place accurately despite the limited size of the MD simulation cell. The genuine MD microfields are directly utilizable in semiclassical linebroadening calculations providing the means to build a clear link between theoretical models and plasma spectroscopy experiments. An example of such a calculation has been reported here for the simple case of electron broadening of a single ion in an electron gas. It shows the magnitude, virtually observable, of the discrepancy induced by the independent quasi-particle approximation. Line profile simulations based on the two component plasma will be a unique way to perform reference data for further developments of fast lineshape models.

\section{Acknowledgments}

Support for this research has been provided by the U. S. Department of Energy grant No. DE-FG03-98DP0218. J. Dufty is grateful for the support and hospitality of the University of Provence.

[1] Minoo H, Gombert M and Deutsch C 1981 Phys. Rev. A 232041

[2] Talin B, Calisti A and Dufty J W 2002 Phys. Rev. E 65, 056406

[3] Dufty J W, Pogorelov I, Calisti A and Talin B this issue.

[4] Pschiwul T and Zwicknagel G 2001 Contrib. Plasma Phys. 41271

[5] Knaup M, Zwicknagel G, Reihard P G, and Toepffer C 2000 J. Phys. France IV10 307 
[6] Rogers F J 1984 Phys. Rev. A 29868

[7] Gigosos M A, Fraile J, and Torres F 1985 Phys RevA 31 3509; Gigosos M A and Cardeñoso v, 1987 J Phys B 20 6005; Gigosos M A and Cardeñoso v, 1996 J Phys B 294795

[8] Collins L, Kwon I, Kress J, Troullier N and Lynch D 1995 Phys. Rev. E 526202

[9] Dharma-Wardana C in Strongly Coupled Plasmas, 275, de Witt H and Rogers F editors (NATO ASI Series, Plenum, NY, 1987). 